\title{
Role of Endosomes and Lysosomes in Human Disease
}

\author{
Frederick R. Maxfield \\ Department of Biochemistry, Weill Cornell Medical College, New York, New York 10065 \\ Correspondence: frmaxfie@med.cornell.edu
}

In addition to their roles in normal cell physiology, endocytic processes play a key role in many diseases. In this review, three diseases are discussed as examples of the role of endocytic processes in disease. The uptake of cholesterol via LDL is central to our understanding of atherosclerosis, and the study of this disease led to many of the key breakthroughs in understanding receptor-mediated endocytosis. Alzheimer's disease is a growing burden as the population ages. Endosomes and lysosomes play important but only partially understood roles in both the formation and the degradation of the amyloid fibrils that are associated with Alzheimer's disease. Inherited lysosomal storage diseases are individually rare, but collectively they affect many individuals. Recent advances are leading to improved enzyme replacement therapy and are also leading to small-molecule drugs to treat some of these diseases.

\begin{abstract}
Endocytosis plays many vital roles in normal Ecell physiology, and as described in this article, endocytic processes can also play significant roles in pathology. Nutrient uptake is one of the essential functions of endocytosis. Two of the best-characterized examples of this are the uptake of cholesterol via the low-density lipoprotein (LDL) receptor (Goldstein and Brown 2009) and the uptake of iron via transferrin and the transferrin receptor (Aisen et al. 2001). Another important role for endocytosis is the regulation of cell-surface expression of membrane proteins, especially receptors and transporters. The balance between recycling or trafficking to storage organelles or to late endosomes and lysosomes (LE/Ly) is often a determining factor in regulating surface expression levels of membrane proteins. Thus, the membrane sort-
\end{abstract}

ing that occurs in endosomes is important for regulating cell physiology. The $\mathrm{pH}$ levels in endosomes play an important role in many functions of endocytosis, including release of iron from transferrin, release of LDL and other ligands from their receptors, and activation of lysosomal hydrolases. As discussed herein, many of these same processes can also play a role in human diseases. A few specific diseases-atherosclerosis, Alzheimer's disease, and lysosomal storage diseases—are used to illustrate this.

\section{ROLE OF ENDOSOME AND LYSOSOME ACIDIFICATION}

A schematic diagram of typical endocytic pathways in a nonpolarized mammalian cell is shown in Figure 1. After internalization by clathrin-

Editors: Sandra L. Schmid, Alexander Sorkin, and Marino Zerial

Additional Perspectives on Endocytosis available at www.cshperspectives.org

Copyright (C) 2014 Cold Spring Harbor Laboratory Press; all rights reserved; doi: 10.1101/cshperspect.a016931

Cite this article as Cold Spring Harb Perspect Biol 2014;6:a016931 
F.R. Maxfield

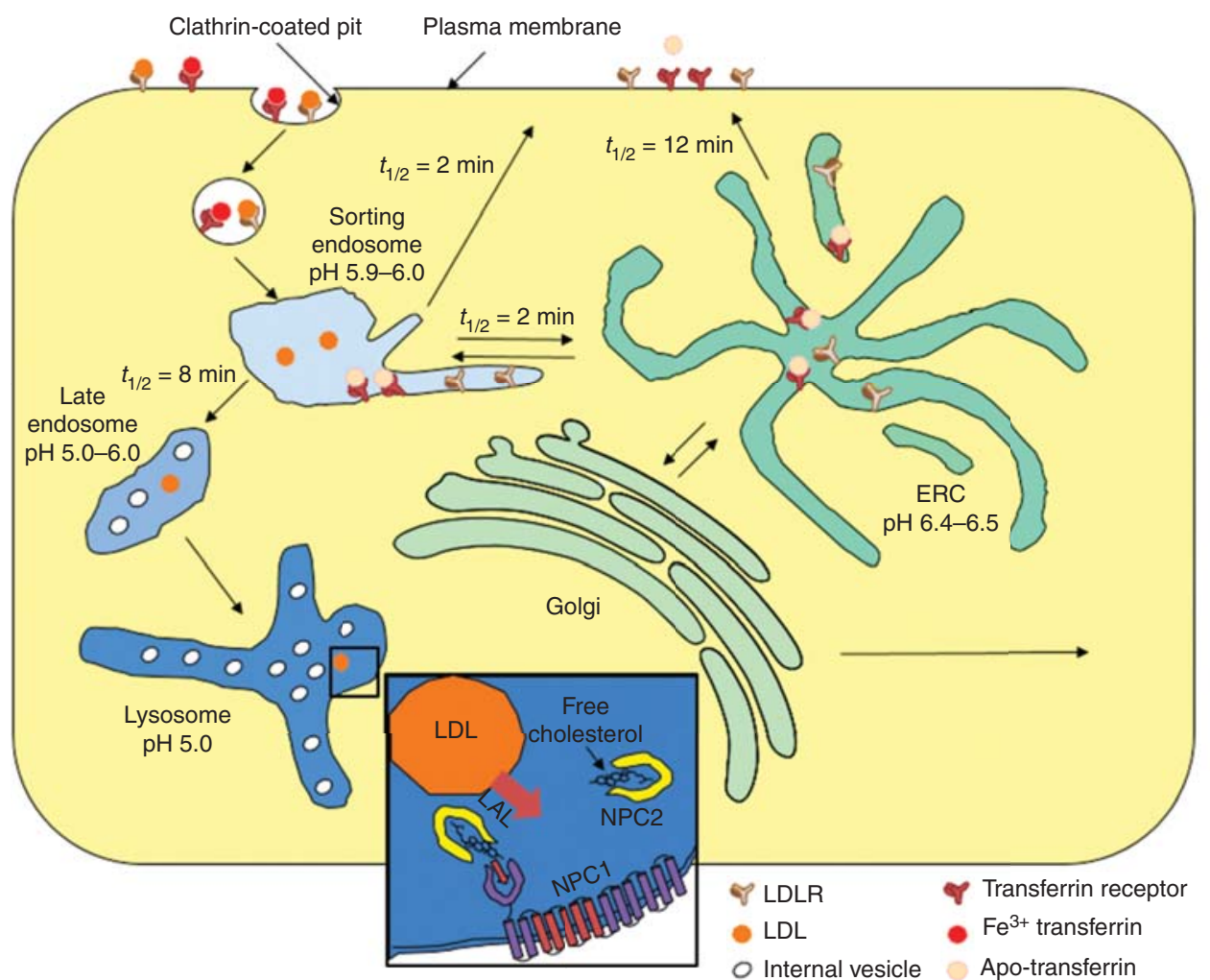

Figure 1. Receptor-mediated endocytic pathway in nonpolarized mammalian cells. The main pathways following clathrin-mediated endocytosis are illustrated. The $\mathrm{pH}$ of various organelles and the kinetics of passage between organelles are shown. LDL and transferrin and their receptors are shown as examples. (Inset) Cholesterol, produced by hydrolysis of cholesteryl esters by lysosomal acid lipase, binds to NPC2 and then NPC1 as part of the mechanism to facilitate cholesterol exit from the digestive organelles.

mediated endocytosis, membrane proteins are delivered to early sorting endosomes, which are tubulovesicular organelles (Geuze et al. 1984; Gruenberg and Maxfield 1995). Other clathrin-independent endocytic processes also deliver membrane proteins into early sorting endosomes (Mayor and Pagano 2007; Howes et al. 2010). The $\mathrm{pH}$ in endosomes has been measured using $\mathrm{pH}$-sensitive fluorescent dyes coupled to ligands that are delivered to these organelles (Dunn et al. 1994). The early sorting endosomes have a $\mathrm{pH}$ of $\sim 5.6-6.0$ (Maxfield and Yamashiro 1987). This low $\mathrm{pH}$ causes transferrin to release its bound iron, which is then transported out of the endosomes (Aisen et al. 2001). The mildly acidic $\mathrm{pH}$ also causes many ligands, including LDL (Rudenko et al. 2002) and insulin (Borden et al. 1990), to dissociate from their receptors, thus uncoupling the trafficking fate of the ligands and receptors (Geuze et al. 1984).

A portion of the membrane in early sorting endosomes returns directly to the plasma membrane by a fast recycling route (Hao and Maxfield 2000; Shah et al. 2013). Most of the remainder of the membrane is removed from the sorting endosome via narrow-diameter tubules and is delivered to the endocytic recycling compartment (ERC), which is also slightly acidic (Yamashiro et al. 1984; Dunn et al. 1989). Inclusion or exclusion of membrane proteins and lipids in these tubules is a major determinant of the efficiency of recycling these membrane components. During the lifetime of a sorting endosome, there are many rounds of removal of membrane, which can lead to highly efficient sorting of membrane constituents on 
a recycling pathway (Dunn et al. 1989; Mukherjee et al. 1997). The narrow diameter of the tubules helps to reduce removal of soluble contents from the sorting endosomes.

The early sorting endosomes stop fusing with newly formed endocytic vesicles after 510 min (Dunn et al. 1989; Dunn and Maxfield 1992), and this begins a process of maturation into late endosomes, which have a somewhat lower internal $\mathrm{pH}$ (5.0-5.5) (Maxfield and Yamashiro 1987; Yamashiro and Maxfield 1987). The late endosomes also receive material delivered from the trans-Golgi network (TGN), including newly synthesized lysosomal hydrolases bound to mannose-6-phosphate receptors (Ghosh et al. 2003; Braulke and Bonifacino 2009). The hydrolases, with their mannose-6phosphate glycoconjugates, are released from the receptors because of the low $\mathrm{pH}$ in the late endosomes, and the empty receptors can recycle back to the TGN. Lysosomal hydrolases generally require an acidic $\mathrm{pH}$ for activity, and the low $\mathrm{pH}$ in the late endosomes activates their enzymatic activity.

Endocytic organelles are acidified through the action of a vacuolar ATPase (V-ATPase), which is a large multimeric protein complex (Toei et al. 2010). The V-ATPase is an electrogenic proton pump, which means that the positive charge from the proton is not balanced by a counter-ion as the proton is transported into the endosomes. This positive charge inside the endosomes will limit the further acidification of the endosomes unless there is transport of another ion to balance the charge transfer of the proton. This counter-ion transport could be accomplished by efflux of cations out of the endosomes and lysosomes (Steinberg et al. 2010) or by transport of anions into the organelles (Ishida et al. 2013). There is evidence that both processes occur, and the $\mathrm{pH}$ of the various endosomes is determined in large part by the activity of these counter-ion transport processes.

\section{ROLE OF ENDOSOMES AND LYSOSOMES IN ATHEROSCLEROSIS}

Most mammalian cells derive cholesterol by a combination of synthesis and uptake via li- poproteins. Dietary cholesterol is absorbed by intestinal epithelia and secreted as chylomicrons, large lipoproteins filled with cholesteryl esters and triglycerides. As the chylomicrons pass through the blood vessels, they are catabolized by lipoprotein lipase, an enzyme that is on the surface of endothelial cells-especially in adipose tissues (Young and Zechner 2013). The released fatty acids are then taken up by cells in the underlying tissue. The chylomicron remnants are cleared from the circulation by hepatocytes, which use the cholesterol and fatty acids to produce very-low-density lipoproteins (VLDLs), which are secreted as large lipoproteins filled with cholesteryl esters and triglycerides. (The chylomicrons contain apolipoprotein B-48, whereas the VLDLs contain apolipoprotein B-100. Both types of particles also have several apoE proteins reversibly associated with them.) Like chylomicrons, VLDL is catabolized by lipoprotein lipase on the surface of endothelial cells. The remaining apoB100containing LDL particles are $22-24 \mathrm{~nm}$ in diameter and have a core that is mainly cholesteryl esters. LDL is the major lipoprotein in human serum, and its role is to deliver cholesterol to peripheral cells. The abundance of LDL in serum is determined in large part by the balance between VLDL secretion from the liver and the uptake of LDL by the liver. Formation of bile salts from cholesterol and secretion from the liver into the digestive tract is the major pathway for elimination of cholesterol from the body.

The synthesis of cholesterol and the expression of LDL receptors are both controlled by the Insig-SCAP-SREBP2 proteins that reside in the endoplasmic reticulum (ER) (Brown and Goldstein 1999). When cholesterol levels in the ER are low, SCAP and SREBP2 are released from Insig, and they enter COPII vesicles for transport to the Golgi. Two proteases in the Golgi cleave the SREBP2 protein, producing a soluble fragment, which is a transcription factor that enters the nucleus and increases the transcription of the enzymes in the cholesterol biosynthetic pathway as well as the expression of LDL receptors. This system is shut off when cholesterol levels are high, leading to a reduction in cholesterol synthesis and uptake. 
F.R. Maxfield

LDL provides a source of cholesterol and fatty acids that cells throughout the body can use for growth of their membranes, and the fatty acids are an energy source. (LDL does not cross the blood-brain barrier, so all cholesterol in the CNS must be synthesized there.) In the classical work of Brown and Goldstein, it was shown that LDL binds to the LDL receptor, which is endocytosed via clathrin-mediated endocytosis (Goldstein and Brown 2009). In late endosomes and lysosomes, cholesteryl esters in the core of the LDL are digested by lysosomal acid lipase, releasing cholesterol and fatty acids. The cholesterol, which is very poorly soluble in water, is transported out of LE/Ly through the action of two proteins (NPC1 and NPC2) that were identified through their association with Niemann Pick disease type C (NPC), an inherited lysosomal storage disease (Carstea et al. 1997; $\mathrm{Xu}$ et al. 2007). The NPC2 protein is a soluble cholesterol-binding protein that is targeted to LE/Ly by a mannose-6-phosphate modification (Liou et al. 2006). The NPC1 protein is a polytopic membrane protein with a cholesterolbinding site in its lumenal amino-terminal domain (Kwon et al. 2009). A current model is that NPC2 binds cholesterol that is produced in LE/Ly by hydrolysis of cholesteryl esters in the core of the internalized LDL. The NPC2 then hands off the cholesterol to the NPC1 amino-terminal domain (Wang et al. 2010; Deffieu and Pfeffer 2011). The cholesterol is then able to leave the LE/Ly, perhaps by insertion into the limiting membrane of the LE/Ly followed by transfer to a cytoplasmic cholesterol-binding protein.

High levels of dietary fat and cholesterol, which are now typical in most economically advanced societies, appear to place this homeostatic mechanism outside of its functional range. Excess cholesterol entering the liver from chylomicron remnants leads to down-regulation of LDL receptors on the surface of hepatocytes. This leads to a reduction in the endocytic clearance of LDL from the circulation, leading to elevated levels of LDL in the blood. Cardiovascular disease, for which elevated LDL is a major risk factor, is the most frequent cause of death in the United States (Minino 2013).
Although metabolism of cholesterol and lipoproteins has been studied intensely for many years, there is still much that we do not understand about these processes. For example, PCSK9, a novel regulator of the surface expression of LDL receptors in hepatocytes, has been identified recently, and its mechanism of action is only partially understood (Horton et al. 2009; Cohen and Hobbs 2013). PCSK9 is a soluble protein that is secreted from hepatocytes. It binds to extracellular domains on the LDL receptor and reduces the efficiency of recycling of the LDL receptor in hepatocytes. This leads to lysosomal degradation of the LDL receptors. PCSK9 was discovered in a screen for people with low levels of LDL. An individual with LDL levels $\sim 10 \%$ of typical values was found to have homozygous mutations in PCSK9 and no detectable circulating PCSK9. Heterozygotes had reduced levels of PCSK9 and reduced levels of LDL. This finding is leading to attempts to reduce PCSK9 levels as a method to increase LDL clearance by the liver and reduced circulating LDL (Cohen and Hobbs 2013).

LDL can percolate through the endothelial lining, and these lipoproteins are then exposed to lipases and other factors that lead to their aggregation, oxidation, and retention in the blood vessel wall (Moore and Tabas 2011). These lipoproteins can be recognized by various receptors on the surface of macrophages, including scavenger receptors that recognize oxidized lipoproteins (Moore and Tabas 2011). Normally, macrophages would internalize these lipoproteins and digest them in late endosomes and lysosomes. The cholesterol can then be stored in the macrophages as cholesteryl esters in lipid droplets or effluxed to HDL particles that are able to carry cholesterol back to the liver (Maxfield and Tabas 2005).

When LDL cholesterol levels are too high, the macrophages become overwhelmed by the excessive uptake of cholesterol. The cells become enlarged, and their cytoplasm becomes filled with cholesteryl ester lipid droplets, giving them a foamy appearance. Eventually, these foam cells undergo ER stress, which leads to cell death (Maxfield and Tabas 2005; Moore and Tabas 2011). The cellular deposits of cholesteryl 
esters are left behind, and additional macrophages are converted to foam cells as they try to clear the remnants of these dead macrophages. The macrophages secrete cytokines that attract additional macrophages into the area, and this inflammatory milieu also leads to proliferation of smooth muscle cells. Progression of this process leads to growth of the atherosclerotic plaque, which bulges into the vessel wall, restricting blood flow. In the worst case, a growing plaque can rupture, leading to formation of a blood clot that fully closes the blood vessel, causing a heart attack or stroke.

The cellular mechanisms for the uptake of LDL-derived cholesterol by macrophages in the vessel wall are under investigation. Because the retained and aggregated LDL is tightly bound to the extracellular matrix, it cannot be taken up by the typical mechanism of binding to a mobile receptor on the cell surface and then entering via coated pits. Even for phagocytosis, the aggregated LDL (agLDL) would need to be released from the extracellular matrix-perhaps by proteolysis of the matrix proteins. An alternative model is that the agLDL is actually digested by lysosomal enzymes outside the cell. When macrophages in a cell culture model contact agLDL, a unique set of cell responses is triggered (Grosheva et al. 2009; Haka et al. 2009, 2013). This results in the formation of an extracellular, acidic compartment, called a surface-connected compartment (Zhang et al. 1997) or a lysosomal synapse, into which lysosomal contents are secreted (Fig. 2). A tight seal is formed between the cell and the agLDL by an actin-dependent process that forms a highly convoluted surface of the cell in the region of contact. Protons are secreted into the lysosomal synapse by the $\mathrm{V}$ ATPase, and the diffusion of the protons is hindered sufficiently within regions of this compartment to allow acidification below $\mathrm{pH} 6$ (Haka et al. 2009). This activates the secreted lysosomal hydrolases, including lysosomal acid lipase, which hydrolyzes the cholesteryl esters, leading to delivery of cholesterol into the macrophage. Eventually, the hydrolases break up the agLDL into small pieces that can be endocytosed and delivered to LE/Ly inside the cell for complete digestion. A macrophage in contact with agLDL can take up a large amount of cholesterol in a short period of time, and the excess cholesterol is rapidly re-esterified and stored as cholesteryl esters in cytoplasmic lipid droplets.

In vivo studies show that the LDL in atherosclerotic lesions is aggregated and tightly associated with the extracellular matrix. There are abundant extracellular lysosomal hydrolases in these lesions as well. However, it has not yet been demonstrated that the hydrolysis of agLDL cholesteryl esters occurs by the same

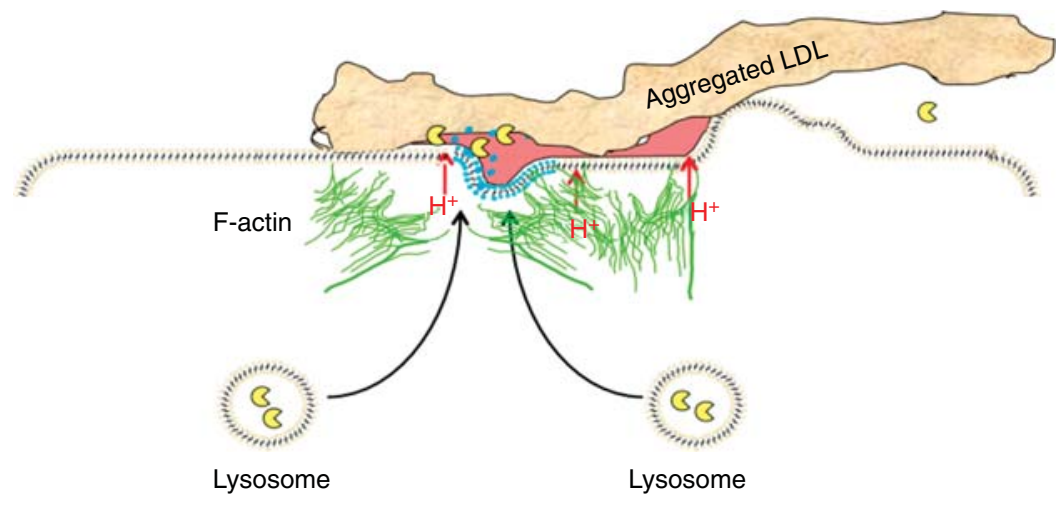

Figure 2. A lysosomal synapse. When a macrophage encounters aggregated LDL in the blood vessel wall, it forms a tight seal using actin-dependent membrane rearrangements. The sealed area is acidified by V-ATPase proton pumps in the plasma membrane, and lysosomes fuse in the contact area. This creates an extracellular, acidic, hydrolytic compartment in which cholesteryl esters in the LDL are digested, and the cholesterol can then be taken up by the macrophage. 
F.R. Maxfield

process that has been demonstrated in cell culture models.

\section{ROLE OF ENDOSOMES AND LYSOSOMES IN ALZHEIMER'S DISEASE}

As life expectancy has increased, Alzheimer's disease $(\mathrm{AD})$ has emerged as a major cause of disability in the elderly. A rough estimate is that there were 26 million people with $\mathrm{AD}$ in 2006, and that the number will quadruple by 2050 (Brookmeyer et al. 2007). Aside from the human cost to those suffering from the disease and their families, the projected financial costs of caring for $\mathrm{AD}$ patients is anticipated to have enormous economic consequences if effective treatments are not developed. A major cause of $\mathrm{AD}$ is various oligomeric and polymeric forms of $A \beta$, a 38- to 43-residue peptide that is formed by two sequential proteolytic cleavages of a transmembrane protein called the amyloid precursor protein (APP) (Thinakaran and Koo 2008).

Newly synthesized APP is a glycoprotein that passes through the ER and the Golgi and is transported to the plasma membrane. At steady state, only a small fraction of APP is found on the surface because it is internalized by clathrin-mediated endocytosis attributed to a YENPTY internalization motif near the carboxyl terminus. Some internalized APP is degraded in LE/Ly. More importantly, three selective proteases can cleave APP, leading to various products. An $\alpha$-secretase cleaves APP at the cell surface within the $A \beta$ peptide sequence. This releases the amino-terminal domain into the extracellular space, and it prevents further processing to form the amyloidogenic $A \beta$ peptide. Neurons express high levels of a $\beta$-secretase that is a transmembrane aspartyl protease called BACE1. BACE1 cleaves APP in the lumenal/ extracellular domain, exposing the amino terminus of $A \beta$. The $\gamma$-secretase cleaves at several sites within the transmembrane domain of APP, releasing $A \beta$ peptides $38-43$ residues in length and a carboxy-terminal fragment. The predominant species released by neurons is $A \beta 40$ with a smaller amount of $A \beta 42$ and very small amounts of the other peptides.
It has been challenging to determine which compartments are responsible for generation of $A \beta$ peptides. APP, BACE1, and the $\gamma$-secretase complex are found throughout the secretory and endocytic pathways (Fig. 3). It is clear that BACE1 cleavage precedes processing by the $\gamma$-secretase, and there is strong evidence that BACE1 cleavage occurs in the early endosome system (sorting endosomes and the ERC), presumably after endocytosis (Huse et al. 2000). The site of $\gamma$-secretase cleavage is somewhat more uncertain. When APP is expressed in HEK293 cells, a series of shRNA knockdown experiments shows that the retromer complex is required for the most efficient cleavage by $\gamma$ secretase, and knockdowns that increase delivery of APP to the TGN increase formation of A $\beta$ peptides (Choy et al. 2012). This would be consistent with most $\gamma$-secretase processing occurring in the TGN after the BACE1-cleaved APP passes from the ERC to the TGN. Other models support a role for the AP4 adaptor complex in $\gamma$-secretase processing in endosomes en route from the TGN to the cell surface (Burgos et al. 2010). It is possible that $\gamma$-secretase processing can occur in more than one organelle.

The $A \beta$ peptide is very prone to aggregation, oligomerization, and formation of $\beta$-sheet fibrils. Although there is a clear association between the excess net formation of $A \beta$ peptide and Alzheimer's dementia in people with some inherited forms of early onset $\mathrm{AD}$, the form of $A \beta$ peptide that is responsible for neurotoxicity remains uncertain (Mucke and Selkoe 2012). A $\beta$ fibrils can associate with each other to form the large amyloid plaques that are one of the hallmarks of the disease, but there is only a partial correlation between plaque abundance at autopsy and dementia in $\mathrm{AD}$ patients. This has led to the hypothesis that it is smaller units of $A \beta$ peptide oligomerization or short fibrils that are the main toxic agent (Selkoe 2012). These small $A \beta$ oligomers or fibrils may begin to form inside neurons (Capetillo-Zarate et al. 2012), but the main site of $A \beta$ fibril accumulation is extracellular.

A $\beta$ peptides are formed in the brain throughout life, so the net accumulation of the neurotoxic forms of $A \beta$ must represent a 


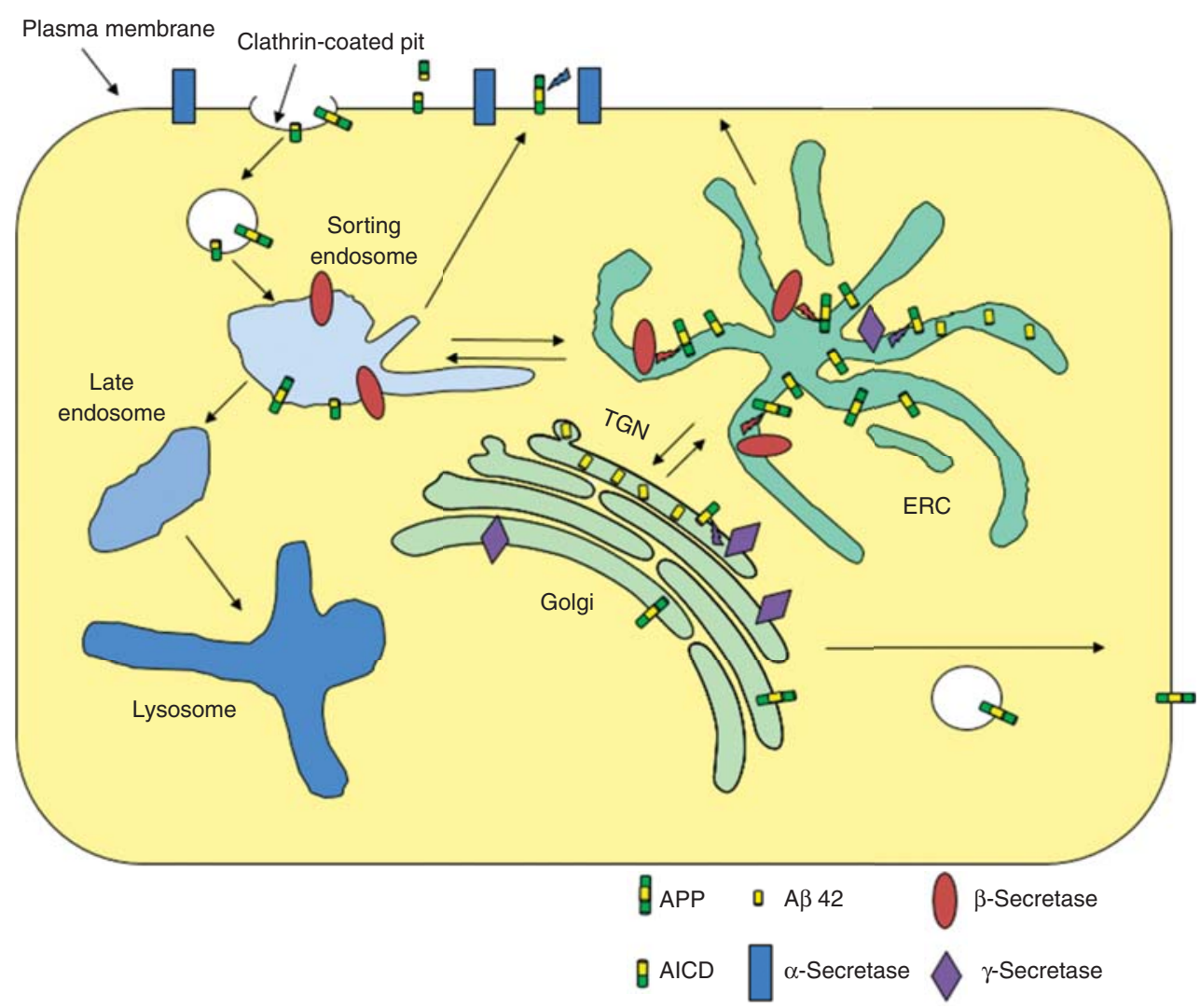

Figure 3. Processing of APP to form A $\beta$ peptide. APP is secreted by a constitutive secretory pathway from the Golgi. While on the plasma membrane, APP can be cleaved by $\alpha$-secretases in the middle of the A $\beta$ peptide region, thus preventing formation of A $\beta$ peptide. APP is internalized by clathrin-mediated endocytosis. In the early endosome system, probably in the ERC, APP can be cleaved by BACE1 to release the amino-terminal domain into the lumen of the endosome. The carboxy-terminal transmembrane fragment can be cleaved by the $\gamma$-secretase within the transmembrane segment, perhaps after retrograde transport back to the TGN. The amyloidogenic $A \beta$ peptide is released into the lumen of the organelles. As discussed in the text, these various steps of proteolytic processing may take place in several different organelles because APP and the various enzymes are found throughout the secretory and endocytic pathways.

balance between production and degradation (Hardy and Selkoe 2002; Blennow et al. 2012). Several enzymes have been identified that can cleave monomeric forms of $A \beta$. The fibrillar forms of $A \beta$ and amyloid plaques are resistant to proteolysis, and it was thought that these might be essentially permanent once formed. However, experiments in mice showed that after immunization against $\mathrm{A} \beta$ peptides, $\mathrm{AD}$ model mice could clear previously formed $A \beta$ plaques (Bard et al. 2000). Microglia, which are found in close proximity to plaques, were proposed to play an important role in the clearance of $A \beta$ fibrils and amyloid plaques (KoenigsknechtTalboo et al. 2008).

Microglia can bind fibrillar forms of $A \beta$ through specific receptors (e.g., scavenger receptor type A) on their surface (Paresce et al. 1996). Small fibrils (average size $\sim 100 \mathrm{~nm}$ in length) can be internalized by receptor-mediated endocytosis and delivered to LE/Ly. In macrophages and other cell types, the fibrillar $A \beta$ is degraded in these organelles (Majumdar et al. 2008). However, in microglia, which are the cells in the brain that clear dead cells and denatured extracellular proteins, the digestion of fibrillar 


\section{F.R. Maxfield}

$\mathrm{A} \beta$ is very slow, with only partial digestion $12 \mathrm{~d}$ after internalization by cultured microglia (Chung et al. 1999). When compared with macrophages, which digest fibrillar $\mathrm{A} \beta$ effectively, microglia were found to have equal or greater levels of several key lysosomal proteases when assayed in cell extracts in acidic buffers (Majumdar et al. 2007). However, the average lysosomal $\mathrm{pH}$ in microglia was $\sim 6.0$ as compared with 5.0 in the macrophages (Majumdar et al. 2007). Because many lysosomal hydrolases require a low $\mathrm{pH}$ for activation and for maximal enzymatic activity, the high $\mathrm{pH}$ in the lysosomes would be expected to dramatically reduce the hydrolytic capabilities in microglia. The cause for the poor acidification of microglial lysosomes is low abundance of a chloride transporter, ClC-7 (Fig. 4). In quiescent cultured microglia, expression of $\mathrm{ClC}-7$ and its heterodimeric partner Ostm 1 are very low as compared with other cell types (Majumdar et al. 2011). Overexpression of ClC-7 and Ostm1 allows further acidification of the lysosomes in microglia, and this is associated with an increased ability to degrade fibrillar $A \beta$ that has been internalized into lysosomes.

When cultured microglia are activated with the inflammatory cytokine macrophage colony stimulating factor (MCSF), they increase the synthesis of ClC-7 and Ostm1 (Majumdar et al. 2011). ClC-7 can then be detected in the lysosomes of the microglia, and the lysosomal pH drops to an average value of 5.0. These MCSFactivated microglia are now able to digest fibrillar A $\beta$. RNAi knockdown of ClC-7 during the activation process impairs the acidification of lysosomes and reduces the ability of the activated microglia to degrade fibrillar $A \beta$. These data show that microglia regulate their lysosomal $\mathrm{pH}$ to a higher level than other cells by restrict-

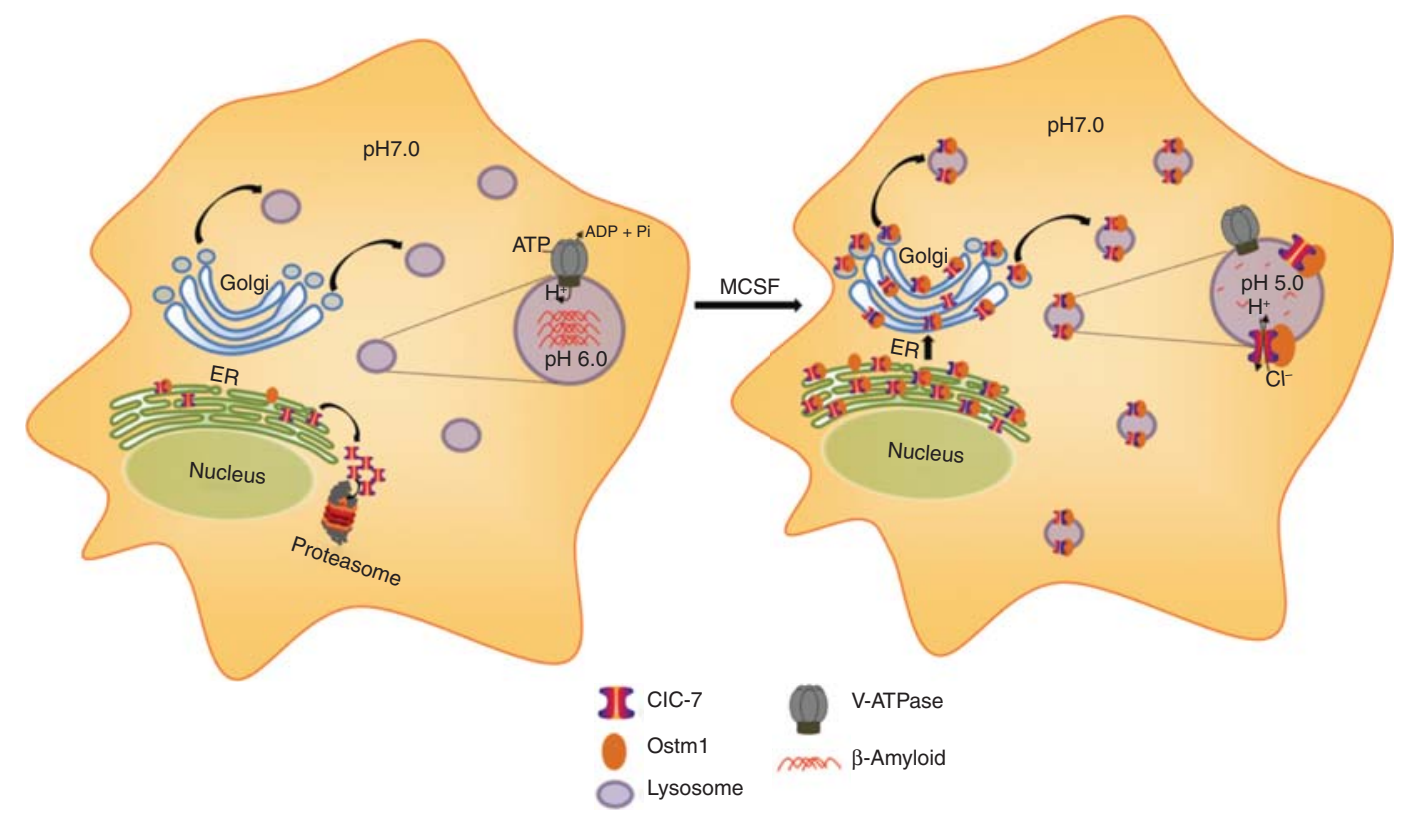

Figure 4. Regulation of lysosomal $\mathrm{pH}$ in microglia. Unlike most cells, quiescent microglia (left) maintain a pH of $\sim 6.0$ in their lysosomes. This limits the hydrolytic activity of the lysosomal acid hydrolases, and there is very little degradation of fibrillar forms of $\mathrm{A} \beta$ in these weakly acidic lysosomes. The ClC-7 chloride transporter plays an important role in dissipating the electrical gradient produced by the proton-pumping V-ATPase. In quiescent microglia, only low levels of $\mathrm{ClC}-7$ and its heterodimeric partner, Ostm1, are expressed, and most of the ClC-7 is degraded by an ER-associated degradation. When microglia are activated by MCSF, expression of both CLC-7 and Ostm 1 is increased, and $\mathrm{ClC}-7$ is delivered to lysosomes. This allows full acidification to $\mathrm{pH}$ 5.0, and internalized fibrillar $\mathrm{A} \beta$ can be digested efficiently. 
ing the expression of $\mathrm{ClC}-7$ and its delivery to lysosomes. This indicates that in these cells, ClC7-dependent chloride transport is required to counterbalance the charge transfer by the electrogenic V-ATPase.

It is unclear what purpose is served by incomplete lysosomal acidification in quiescent microglia. The average $\mathrm{pH}$ of 6.0 is sufficient to allow degradation of many other internalized proteins, although degradation is presumably slower than in other cell types. The proteaseresistant $\beta$-sheets of the $A \beta$ fibrils seem to be particularly resistant to degradation when the $\mathrm{pH}$ is not optimal. Microglia are the main antigen-presenting cells of the central nervous system (CNS), and it is possible that a slower lysosomal degradation process is useful for preserving potential antigens as has been proposed for dendritic cells (Trombetta et al. 2003; Jancic et al. 2007). Upon inflammatory activation, the microglia can fully acidify their lysosomes and degrade fibrillar A $\beta$ effectively (Majumdar et al. 2011), and this may account for the degradation of amyloid plaques following immunization (Koenigsknecht-Talboo et al. 2008).

In $\mathrm{AD}$ model mice, it has been shown that inflammatory stimuli in the brain lead to clearance of amyloid plaque, and there is evidence that this is associated with cognitive improvements (Boissonneault et al. 2009; Yong and Rivest 2009). Much more work is required to determine how lysosomal degradation affects the balance between production and removal of $A \beta$ and how this relates to the neuropathology of AD. However, it is possible that non-inflammatory activation of lysosomal functions could be beneficial.

\section{LYSOSOMAL STORAGE DISEASES AND MEMBRANE TRAFFIC}

The lysosomal storage diseases are the classic example of a disease of the endosome/lysosome system (Walkley 1998). Most of these disorders are due to mutations in a gene coding for an individual lysosomal hydrolase or another protein that participates in the hydrolytic mechanism (e.g., a saposin) (Kolter and Sandhoff 2005). The substrate for the defective enzyme then accumulates in the lysosomes. The diseases are generally recessive, and heterozygotes are typically asymptomatic. Individually, these diseases are quite rare, but as a class the lysosomal storage diseases have been estimated to affect one in 7700 live births among people of European ancestry (Meikle et al. 1999). More recent studies suggest that this is a serious underestimate because four diseases (Fabry, Gaucher, Pompe, and Niemann Pick A and B) showed an incidence of 1:2315 in an Austrian population based on enzyme assays followed by sequencing studies (Mechtler et al. 2012).

It is often not clear precisely why the accumulated substrates for the missing enzymes cause pathology. Additionally, the most severe effects of the storage defects can affect only certain organs or even specific regions of the brain while leaving other tissues or brain regions largely unaffected even if the storage is readily apparent (Walkley 1998). It is often noted that the storage defects are associated with changes in other aspects of membrane trafficking and cell physiology for which the connection to substrate accumulation in LE/Ly is not obvious.

A fraction of the mannose-6-phosphate receptors are found on the plasma membrane, and these receptors can bind mannose-6-phosphate modified proteins and endocytose them via clathrin-mediated endocytosis. The ligands are released upon exposure to the low $\mathrm{pH}$ in endosomes, and the receptors can recycle back to the plasma membrane or be returned to the TGN (Tortorella et al. 2007). This surface pool may normally serve to capture enzymes that are secreted rather than being delivered via the predominant intracellular route to LE. Therapeutically, this uptake of extracellular mannose-6phosphate ligands allows the uptake of enzymes to correct enzyme deficiencies. Following demonstration of the effectiveness of this strategy in cell culture (Rome et al. 1979) and animal models, there have now been several successful implementations of this strategy in human patients. These include enzyme replacement therapy for $\beta$-glucocerebrosidase (Gaucher disease) (Mistry et al. 2011), some forms of mucopolysaccharidosis (de Ru et al. 2011), $\alpha$-galactosidase A (Fabry disease) (Pisani et al. 2011), 
F.R. Maxfield

acid $\alpha$-glucosidase (Pompe disease) (Angelini and Semplicini 2012), and others that are being tested (e.g., acid sphingomyelinase deficiency) (Schuchman 2009).

There are several challenges associated with enzyme replacement therapy. One is that the enzymes have a lifetime of only about a day or two in the LE/Ly, so the process must be repeated frequently enough to prevent toxic accumulation of substrates. There can be immune responses to the enzymes (Angelini and Semplicini 2012), but in some cases, patients can become immune-tolerant to the enzymes (Kakavanos et al. 2003). A major challenge is delivering enzyme to the CNS when the disease involves neuropathology - as it often does. There are examples of intrathecal injections to deliver enzymes, and delivery to the brain is being explored (Lachmann 2011). Because the effects of these diseases can be so devastating, these expensive and difficult therapeutic approaches are approved by regulatory agencies and tolerated by patients and their families.

An alternative to injection of enzymes is gene therapy in which a fraction of cells are transfected, but these cells can produce large amounts of enzyme that is secreted (Sondhi et al. 2012). The secreted enzyme can then be internalized by other cells, so transfection of only a small fraction of cells can be sufficient for effective therapy.

For some lysosomal storage disorders, small-molecule therapies are being developed. One such strategy involves reduction in the abundance of the substrate for the defective enzyme. An example of this is Miglustat ( $N$-butyldeoxynojirimycin), which is an imino sugar that inhibits glucosylceramide synthase, the first committed step in glycosphingolipid synthesis. Miglustat has been used for treatment of Gaucher disease (Kuter et al. 2013).

In some cases, the mutation in lysosomal enzymes leads to production of a protein that contains a point mutation that impairs its folding but would not block function if the protein could be delivered to the LE/Ly. These mutant proteins fail to pass through the ER quality control and are degraded by an endoplasmic reticulum-associated degradation (ERAD) process.
Reversible competitive enzyme inhibitors that bind to the active site of the enzyme can stabilize the correctly folded form of the protein so that it can escape the ER and be delivered to LE/Ly. These chemical chaperones have been shown to be effective in cell culture, and in some cases in patients (Suzuki 2013). These small-molecule chaperone therapies are particularly appealing for enzyme deficiencies with neuropathology because of the difficulty of enzyme replacement therapy in the CNS.

\section{Niemann Pick C Disease}

Niemann Pick disease type C (NPC) leads to abnormal accumulation of cholesterol in LE/ Ly, and other lipids also accumulate in the storage organelles - apparently as a response to the increased concentration of cholesterol in the internal membranes of these organelles (Vanier 2010). Mutations in NPC1 and NPC2 have been associated with NPC disease, and 95\% of patients have mutations in NPC1. The role of NPC1 and NPC2 has been described in a preceding section.

Miglustat has been used therapeutically for NPC disease, and it slows the progression of the disease (Patterson et al. 2007). The proposed mechanism is that in the brain, gangliosides accumulate to very high levels in the storage organelles. Miglustat reduces synthesis of gangliosides, so it can reduce this lipid accumulation. Because cholesterol has thermodynamically favorable interactions with glycosphingolipids, reducing the ganglioside levels may also reduce the cholesterol storage in these organelles. Consistent with this mechanism, increased acid sphingomyelinase activity decreases the cholesterol accumulation in cultured NPC cells (Devlin et al. 2010). Miglustat has been approved for treatment of NPC disease in the European Union and several other countries but not in the United States.

Most of the mutations in the NPC1 protein are missense mutations leading to single amino acid substitutions (Vanier 2010). In fibroblasts expressing the most frequent mutation,

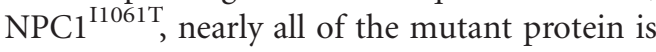
degraded by an ERAD process (Gelsthorpe et al. 
2008). (A high fraction of the wild-type protein is also degraded rapidly.) Overexpression of the mutant protein could partially restore normal cholesterol trafficking, suggesting that the mutant protein retained some function (Gelsthorpe et al. 2008).

In a screen, it was found that histone deacetylase (HDAC) inhibitors could correct the NPC phenotype in cells with the NPC $1^{11061 \mathrm{~T}}$ mutant (Pipalia et al. 2011). It was also found that this was accompanied by increased expression of the $\mathrm{NPC1}^{\mathrm{I} 1061 \mathrm{~T}}$ protein and by its correct delivery to LE/Ly containing LDL. Further preliminary studies have shown that HDAC inhibitors can restore cholesterol trafficking to a varying degree in several different patient fibroblasts expressing several different NPC1 mutations (Pipalia and Maxfield 2011). It appears that the HDAC inhibitors are partially protecting the mutations against degradation in the ER, allowing some of these proteins to be targeted correctly to the LE/Ly, where they maintain some function. Subsequently, it was found that Gaucher fibroblasts with the N370S or L444P mutations in $\beta$-glucocerebrosidase could also be rescued by HDAC inhibition by assisting folding in the ER ( $\mathrm{Lu}$ et al. 2011), indicating that this may be a more general strategy for restoring function for mutant lysosomal proteins that could be at least partially functional if delivered to the LE/Ly. It should be noted that HDAC inhibitors were shown previously to be able to rescue some mutants of the CFTR protein that also undergo ERAD as a consequence of their mutations (Hutt et al. 2010).

\section{CONCLUDING REMARKS AND FUTURE DIRECTIONS}

This article has discussed three of the many diseases that are associated with endocytosis. Each of these has been studied intensely by many investigators, and collectively we have made enormous progress in our understanding. Nevertheless, we have much more to learn even for these well-studied diseases. Better understanding of membrane trafficking has the potential to lead to improved therapies for these and many other disorders.
In the case of atherosclerosis, key early support for the benefits of controlling LDL cholesterol came from the work of Michael Brown and Joseph Goldstein on the endocytic uptake of LDL in the 1970s. However, even with cholesterol-lowering statin therapies, cardiovascular disease remains a major cause of death in the developed world. We are just starting to understand the mechanisms by which PCSK9 regulates hepatic LDL receptors, and we are just beginning to understand how cholesterol is taken up by macrophages in the walls of blood vessels.

Alzheimer's disease is increasing dramatically in prevalence as the world population ages. We now understand key steps in the formation of $A \beta$ peptides, but the mechanisms by which multimeric forms of $A \beta$ cause neuropathology are not clear. We understand some aspects of the clearance of fibrillar $A \beta$ by microglia, but we don't even know if enhancing such clearance would be beneficial.

Lysosomal storage diseases are the prototypical example of a disease of endosomes and lysosomes. With increased gene sequencing and more sensitive tests, we are now recognizing that these diseases are much more common than previously believed. Fortunately, the endocytic pathway provides a means of correcting some of these disorders by enzyme replacement therapy. The blood-brain barrier is a major obstacle to these therapies, and devising methods to bring proteins across the blood-brain barrier is a major research goal. For many of these genetic diseases, missense mutations lead to partially functional proteins with reduced ability to fold in the ER. Various strategies to allow these proteins to avoid ER-associated degradation are being developed and may lead to orally available therapies that can correct defects in the brain and in the peripheral organs.

In this brief review, only a few diseases related to endocytic processes could be discussed, but many of the same endocytic mechanisms play an important role in diseases discussed in other articles in this collection. Membrane trafficking and control of organelle $\mathrm{pH}$ play an important role in the pathophysiology associated with the uptake of viruses and bacteria (see Cossart and Helenius 2014). A fascinating re- 
F.R. Maxfield

cent discovery that links these fields is the demonstration that NPC1 is an intracellular receptor for the Ebola virus and helps to traffic it to LE/Ly as part of the viral entry process (Miller et al. 2012). We are just starting to appreciate how defects in the basic endocytic machinery are associated with numerous other diseases through their effects on the cellular distribution of lipids and proteins involved in signaling and organelle identity (see Di Fiore and von Zastrow 2014).

We still need to know much more about the basic cell biology of endocytic trafficking in order to properly understand many diseases. For example, trafficking from endosomes back to the Golgi is important for processing of APP, as discussed above, and it is also essential for the activity of many bacterial toxins, which often must get back to the ER. However, we have only a partial understanding of how this sorting is achieved and how it is regulated. From the beginning, research into membrane traffic has been spurred by trying to understand disease processes, and in many cases, this disease-related research has led to fundamental cell biology insights. In turn, the understanding of cellular mechanisms has led to novel understanding of disease processes and in some cases to new therapies (see Mellman and Yarden 2013). We can expect this dynamic interplay between basic science and disease-targeted research to continue to yield new discoveries and new therapies for many years.

\section{ACKNOWLEDGMENTS}

I am grateful to Dana Cruz, David Iaea, and Rajesh Singh for assistance with the figures. This research is supported by grants R37DK 27083 and R01HL093324 from the National Institutes of Health and a grant from the Ara Parseghian Medical Research Foundation.

\section{REFERENCES}

${ }^{*}$ Reference is also in this collection.

Aisen P, Enns C, Wessling-Resnick M. 2001. Chemistry and biology of eukaryotic iron metabolism. Int J Biochem Cell Biol 33: 940-959.
Angelini C, Semplicini C. 2012. Enzyme replacement therapy for Pompe Disease. Curr Neurol Neurosci Rep 12: $70-$ 75.

Bard F, Cannon C, Barbour R, Burke RL, Games D, Grajeda H, Guido T, Hu K, Huang J, Johnson-Wood K, et al. 2000. Peripherally administered antibodies against amyloid $\beta$ peptide enter the central nervous system and reduce pathology in a mouse model of Alzheimer disease. Nat Med 6: $916-919$.

Blennow K, Zetterberg H, Fagan AM. 2012. Fluid biomarkers in Alzheimer disease. Cold Spring Harb Perspect Med 2: a006221.

Boissonneault V, Filali M, Lessard M, Relton J, Wong G, Rivest S. 2009. Powerful beneficial effects of macrophage colony-stimulating factor on $\beta$-amyloid deposition and cognitive impairment in Alzheimer's disease. Brain 132: 1078-1092.

Borden LA, Einstein R, Gabel CA, Maxfield FR. 1990. Acidification-dependent dissociation of endocytosed insulin precedes that of endocytosed proteins bearing the mannose 6-phosphate recognition marker. J Biol Chem 265: 8497-8504.

Braulke T, Bonifacino JS. 2009. Sorting of lysosomal proteins. Biochim Biophys Acta 1793: 605-614.

Brookmeyer R, Johnson E, Ziegler-Graham K, Arrighi HM. 2007. Forecasting the global burden of Alzheimer's disease. Alzheimers Dement 3: 186-191.

Brown MS, Goldstein JL. 1999. A proteolytic pathway that controls the cholesterol content of membranes, cells, and blood. Proc Natl Acad Sci 96: 11041-11048.

Burgos PV, Mardones GA, Rojas AL, daSilva LL, Prabhu Y, Hurley JH, Bonifacino JS. 2010. Sorting of the Alzheimer's disease amyloid precursor protein mediated by the AP-4 complex. Dev Cell 18: 425-436.

Capetillo-Zarate E, Gracia L, Tampellini D, Gouras GK. 2012. Intraneuronal $A \beta$ accumulation, amyloid plaques, and synapse pathology in Alzheimer's disease. Neurodegener Dis 10: 56-59.

Carstea EDM, Coleman KG, Loftus SK, Zhang D, Cummings C, Gu J, Rosenfeld MA, Pavan WJ, Krizman DB, Nagle J, et al. 1997. Niemann-Pick C1 disease gene: Homology to mediators of cholesterol homeostasis. Science 277: $228-231$

Choy RW, Cheng Z, Schekman R. 2012. Amyloid precursor protein (APP) traffics from the cell surface via endosomes for amyloid $\beta$ (A $\beta)$ production in the trans-Golgi network. Proc Natl Acad Sci 109: E2077-2082.

Chung H, Brazil MI, Soe TT, Maxfield FR. 1999. Uptake, degradation, and release of fibrillar and soluble forms of Alzheimer's amyloid $\beta$-peptide by microglial cells. J Biol Chem 274: 32301-32308.

Cohen JC, Hobbs HH. 2013. Simple genetics for a complex disease. Science 340: 689-690.

* Cossart P, Helenius A. 2014. Endocytosis of viruses and bacteria. Cold Spring Harb Perpsect Biol doi: 10.1101/ cshperspect.a016972.

Deffieu MS, Pfeffer SR. 2011. Niemann-Pick type C 1 function requires lumenal domain residues that mediate cholesterol-dependent NPC2 binding. Proc Natl Acad Sci 108: $18932-18936$. 
de Ru MH, Boelens JJ, Das AM, Jones SA, van der Lee JH, Mahlaoui N, Mengel E, Offringa M, O’Meara A, Parini R, et al. 2011. Enzyme replacement therapy and/or hematopoietic stem cell transplantation at diagnosis in patients with mucopolysaccharidosis type I: Results of a European consensus procedure. Orphanet J Rare Dis 6: 55.

Devlin C, Pipalia NH, Liao X, Schuchman EH, Maxfield FR, Tabas I. 2010. Improvement in lipid and protein trafficking in Niemann-Pick $\mathrm{C} 1$ cells by correction of a secondary enzyme defect. Traffic 11: 601-615.

* Di Fiore PP, von Zastrow M. 2014. Endocytosis, signaling, and beyond. Cold Spring Harb Perpsect Biol doi: 10.1101/ cshperspect.a016865.

Dunn KW, Maxfield FR. 1992. Delivery of ligands from sorting endosomes to late endosomes occurs by maturation of sorting endosomes. J Cell Biol 117: 301-310.

Dunn KW, McGraw TE, Maxfield FR. 1989. Iterative fractionation of recycling receptors from lysosomally destined ligands in an early sorting endosome. J Cell Biol 109: 3303-3314.

Dunn KW, Mayor S, Myers JN, Maxfield FR. 1994. Applications of ratio fluorescence microscopy in the study of cell physiology. FASEB J 8: 573-582.

Gelsthorpe ME, Baumann N, Millard E, Gale SE, Langmade SJ, Schaffer JE, Ory DS. 2008. Niemann-Pick type C1 I1061T mutant encodes a functional protein that is selected for endoplasmic reticulum-associated degradation due to protein misfolding. J Biol Chem 283: 8229-8236.

Geuze HJ, Slot JW, Strous GJ, Peppard J, von Figura K, Hasilik A, Schwartz AL. 1984. Intracellular receptor sorting during endocytosis: Comparative immunoelectron microscopy of multiple receptors in rat liver. Cell 37: 195-204.

Ghosh P, Dahms NM, Kornfeld S. 2003. Mannose 6-phosphate receptors: New twists in the tale. Nat Rev Mol Cell Biol 4: 202-212.

Goldstein JL, Brown MS. 2009. The LDL receptor. Arterioscler Thromb Vasc Biol 29: 431-438.

Grosheva I, Haka AS, Qin C, Pierini LM, Maxfield FR. 2009. Aggregated LDL in contact with macrophages induces local increases in free cholesterol levels that regulate local actin polymerization. Arterioscler Thromb Vasc Biol 29: 1615-1621.

Gruenberg J, Maxfield FR. 1995. Membrane transport in the endocytic pathway. Curr Opin Cell Biol 7: 552-563.

Haka AS, Grosheva I, Chiang E, Buxbaum AR, Baird BA, Pierini LM, Maxfield FR. 2009. Macrophages create an acidic extracellular hydrolytic compartment to digest aggregated lipoproteins. Mol Biol Cell 20: 4932-4940.

Haka AS, Grosheva I, Singh RK, Maxfield FR. 2013. Plasmin promotes foam cell formation by increasing macrophage catabolism of aggregated low-density lipoprotein. Arterioscler Thromb Vasc Biol 33: 1768-1778.

Hao M, Maxfield FR. 2000. Characterization of rapid membrane internalization and recycling. J Biol Chem 275: 15279-15286.

Hardy J, Selkoe DJ. 2002. The amyloid hypothesis of Alzheimer's disease: Progress and problems on the road to therapeutics. Science 297: 353-356.
Horton JD, Cohen JC, Hobbs HH. 2009. PCSK9: A convertase that coordinates LDL catabolism. J Lipid Res 50: S172-S177.

Howes MT, Mayor S, Parton RG. 2010. Molecules, mechanisms, and cellular roles of clathrin-independent endocytosis. Curr Opin Cell Biol 22: 519-527.

Huse JT, Pijak DS, Leslie GJ, Lee VM, Doms RW. 2000. Maturation and endosomal targeting of $\beta$-site amyloid precursor protein-cleaving enzyme. The Alzheimer's disease $\beta$-secretase. J Biol Chem 275: 33729-33737.

Hutt DM, Herman D, Rodrigues AP, Noel S, Pilewski JM, Matteson J, Hoch B, Kellner W, Kelly JW, Schmidt A, et al. 2010. Reduced histone deacetylase 7 activity restores function to misfolded CFTR in cystic fibrosis. Nat Chem Biol 6: 25-33.

Ishida Y, Nayak S, Mindell JA, Grabe M. 2013. A model of lysosomal pH regulation. J Gen Physiol 141: 705-720.

Jancic C, Savina A, Wasmeier C, Tolmachova T, El-Benna J, Dang PM, Pascolo S, Gougerot-Pocidalo MA, Raposo G, Seabra MC, et al. 2007. Rab27a regulates phagosomal pH and NADPH oxidase recruitment to dendritic cell phagosomes. Nat Cell Biol 9: 367-378.

Kakavanos R, Turner CT, Hopwood JJ, Kakkis ED, Brooks DA. 2003. Immune tolerance after long-term enzymereplacement therapy among patients who have mucopolysaccharidosis I. Lancet 361: 1608-1613.

Koenigsknecht-Talboo J, Meyer-Luehmann M, Parsadanian M, Garcia-Alloza M, Finn MB, Hyman BT, Bacskai BJ, Holtzman DM. 2008. Rapid microglial response around amyloid pathology after systemic anti-A $\beta$ antibody administration in PDAPP mice. J Neurosci 28: 1415614164.

Kolter T, Sandhoff K. 2005. Principles of lysosomal membrane digestion: Stimulation of sphingolipid degradation by sphingolipid activator proteins and anionic lysosomal lipids. Annu Rev Cell Dev Biol 21: 81-103.

Kuter DJ, Mehta A, Hollak CE, Giraldo P, Hughes D, Belmatoug N, Brand M, Muller A, Schaaf B, Giorgino R, et al. 2013. Miglustat therapy in type 1 Gaucher disease: Clinical and safety outcomes in a multicenter retrospective cohort study. Blood Cells Mol Dis 51: 116-124.

Kwon HJ, Abi-Mosleh L, Wang ML, Deisenhofer J, Goldstein JL, Brown MS, Infante RE. 2009. Structure of Nterminal domain of NPC1 reveals distinct subdomains for binding and transfer of cholesterol. Cell 137: 12131224.

Lachmann RH. 2011. Enzyme replacement therapy for lysosomal storage diseases. Curr Opin Pediatr 23: 588-593.

Liou HL, Dixit SS, Xu S, Tint GS, Stock AM, Lobel P. 2006. NPC2, the protein deficient in Niemann-Pick C2 disease, consists of multiple glycoforms that bind a variety of sterols. J Biol Chem 281: 36710-36723.

Lu J, Yang C, Chen M, Ye DY, Lonser RR, Brady RO, Zhuang Z. 2011. Histone deacetylase inhibitors prevent the degradation and restore the activity of glucocerebrosidase in Gaucher disease. Proc Natl Acad Sci 108: 21200-21205.

Majumdar A, Cruz D, Asamoah N, Buxbaum A, Sohar I, Lobel P, Maxfield FR. 2007. Activation of microglia acidifies lysosomes and leads to degradation of Alzheimer amyloid fibrils. Mol Biol Cell 18: 1490-1496. 
F.R. Maxfield

Majumdar A, Chung H, Dolios G, Wang R, Asamoah N, Lobel P, Maxfield FR. 2008. Degradation of fibrillar forms of Alzheimer's amyloid $\beta$-peptide by macrophages. Neurobiol Aging 29: 707-715.

Majumdar A, Capetillo-Zarate E, Cruz D, Gouras GK, Maxfield FR. 2011. Degradation of Alzheimer's amyloid fibrils by microglia requires delivery of $\mathrm{ClC}-7$ to lysosomes. Mol Biol Cell 22: 1664-1676.

Maxfield FR, Tabas I. 2005. Role of cholesterol and lipid organization in disease. Nature 438: 612-621.

Maxfield FR, Yamashiro DJ. 1987. Endosome acidification and the pathways of receptor-mediated endocytosis. $A d v$ Exp Med Biol 225: 189-198.

Mayor S, Pagano RE. 2007. Pathways of clathrin-independent endocytosis. Nat Rev Mol Cell Biol 8: 603-612.

Mechtler TP, Stary S, Metz TF, De Jesus VR, Greber-Platzer S, Pollak A, Herkner KR, Streubel B, Kasper DC. 2012. Neonatal screening for lysosomal storage disorders: Feasibility and incidence from a nationwide study in Austria. Lancet 379: 335-341.

Meikle PJ, Hopwood JJ, Clague AE, Carey WF. 1999. Prevalence of lysosomal storage disorders. JAMA 281: 249 254.

* Mellman I, Yarden Y. 2013. Endocytosis and cancer. Cold Spring Harb Perpsect Biol 5: a016949.

Miller EH, Obernosterer G, Raaben M, Herbert AS, Deffieu MS, Krishnan A, Ndungo E, Sandesara RG, Carette JE, Kuehne AI, et al. 2012. Ebola virus entry requires the host-programmed recognition of an intracellular receptor. EMBO J 31: 1947-1960.

Minino AM. 2013. Death in the United States, 2011. NCHS Data Brief 115: 1-8.

Mistry PK, Cappellini MD, Lukina E, Ozsan H, Mach Pascual S, Rosenbaum H, Helena Solano M, Spigelman Z, Villarrubia J, Watman NP, et al. 2011. A reappraisal of Gaucher disease-diagnosis and disease management algorithms. Am J Hematol 86: 110-115.

Moore KJ, Tabas I. 2011. Macrophages in the pathogenesis of atherosclerosis. Cell 145: 341-355.

Mucke L, Selkoe DJ. 2012. Neurotoxicity of amyloid $\beta$-protein: Synaptic and network dysfunction. Cold Spring Harb Perspect Med 2: a006338.

Mukherjee S, Ghosh RN, Maxfield FR. 1997. Endocytosis. Physiol Rev 77: 759-803.

Paresce DM, Ghosh RN, Maxfield FR. 1996. Microglial cells internalize aggregates of the Alzheimer's disease amyloid $\beta$-protein via a scavenger receptor. Neuron 17: $553-$ 565.

Patterson MC, Vecchio D, Prady H, Abel L, Wraith JE. 2007. Miglustat for treatment of Niemann-Pick C disease: A randomised controlled study. Lancet Neurol 6: 765-772.

Pipalia NH, Maxfield FR. 2011. Investigating the therapeutic potential of HDAC inhibitors for the treatment of Niemann Pick type C1 disease. Mol Biol Cell 22: abstract \#1642. http://niemannpick.nd.edu/assets/52702/all_ abstracts.pdf.

Pipalia NH, Cosner CC, Huang A, Chatterjee A, Bourbon P, Farley N, Helquist P, Wiest O, Maxfield FR. 2011. Histone deacetylase inhibitor treatment dramatically reduces cholesterol accumulation in Niemann-Pick type C1 mu- tant human fibroblasts. Proc Natl Acad Sci 108: 56205625.

Pisani A, Visciano B, Roux GD, Sabbatini M, Porto C, Parenti G, Imbriaco M. 2011. Enzyme replacement therapy in patients with Fabry disease: State of the art and review of the literature. Mol Genet Metab 107: 267-275.

Rome LH, Weissmann B, Neufeld EF. 1979. Direct demonstration of binding of a lysosomal enzyme, $\alpha$-L-iduronidase, to receptors on cultured fibroblasts. Proc Natl Acad Sci 76: 2331-2334.

Rudenko G, Henry L, Henderson K, Ichtchenko K, Brown MS, Goldstein JL, Deisenhofer J. 2002. Structure of the LDL receptor extracellular domain at endosomal $\mathrm{pH}$. Science 298: 2353-2358.

Schuchman EH. 2009. The pathogenesis and treatment of acid sphingomyelinase-deficient Niemann-Pick disease. Int J Clin Pharmacol Ther 47 (Suppl 1): S48-S57.

Selkoe DJ. 2012. Alzheimer's disease. Cold Spring Harb Perspect Biol 3: a004457.

Shah M, Baterina OY Jr, Taupin V, Farquhar MG. 2013. ARH directs megalin to the endocytic recycling compartment to regulate its proteolysis and gene expression. J Cell Biol 202: $113-127$.

Sondhi D, Johnson L, Purpura K, Monette S, Souweidane MM, Kaplitt MG, Kosofsky B, Yohay K, Ballon D, Dyke J, et al. 2012. Long-term expression and safety of administration of AAVrh.10hCLN2 to the brain of rats and nonhuman primates for the treatment of late infantile neuronal ceroid lipofuscinosis. Hum Gene Ther Methods 23: 324-335.

Steinberg BE, Huynh KK, Brodovitch A, Jabs S, Stauber T, Jentsch TJ, Grinstein S. 2010. A cation counterflux supports lysosomal acidification. J Cell Biol 189: 1171-1186.

Suzuki Y. 2013. Chaperone therapy update: Fabry disease, GM1-gangliosidosis and Gaucher disease. Brain Dev 35: 515-523.

Thinakaran G, Koo EH. 2008. Amyloid precursor protein trafficking, processing, and function. J Biol Chem 283: 29615-29619.

Toei M, Saum R, Forgac M. 2010. Regulation and isoform function of the V-ATPases. Biochemistry 49: 4715-4723.

Tortorella LL, Schapiro FB, Maxfield FR. 2007. Role of an acidic cluster/dileucine motif in cation-independent mannose 6-phosphate receptor traffic. Traffic 8: $402-$ 413.

Trombetta ES, Ebersold M, Garrett W, Pypaert M, Mellman I. 2003. Activation of lysosomal function during dendritic cell maturation. Science 299: 1400-1403.

Vanier MT. 2010. Niemann-Pick disease type C. Orphanet J Rare Dis 5: 16.

Walkley SU. 1998. Cellular pathology of lysosomal storage disorders. Brain Pathol 8: 175-193.

Wang ML, Motamed M, Infante RE, Abi-Mosleh L, Kwon HJ, Brown MS, Goldstein JL. 2010. Identification of surface residues on Niemann-Pick C2 essential for hydrophobic handoff of cholesterol to NPC1 in lysosomes. Cell Metab 12: 166-173.

Xu S, Benoff B, Liou HL, Lobel P, Stock AM. 2007. Structural basis of sterol binding by NPC2, a lysosomal protein deficient in Niemann-Pick type C2 disease. J Biol Chem 282: $23525-23531$. 
Yamashiro DJ, Maxfield FR. 1987. Kinetics of endosome acidification in mutant and wild-type Chinese hamster ovary cells. J Cell Biol 105: 2713-2721.

Yamashiro DJ, Tycko B, Fluss SR, Maxfield FR. 1984. Segregation of transferrin to a mildly acidic ( $\mathrm{pH}$ 6.5) paraGolgi compartment in the recycling pathway. Cell 37: $789-800$.

Yong VW, Rivest S. 2009. Taking advantage of the systemic immune system to cure brain diseases. Neuron 64: 55-60.
Young SG, Zechner R. 2013. Biochemistry and pathophysiology of intravascular and intracellular lipolysis. Genes Dev 27: 459-484.

Zhang WY, Gaynor PM, Kruth HS. 1997. Aggregated low density lipoprotein induces and enters surfaceconnected compartments of human monocyte-macrophages. Uptake occurs independently of the low density lipoprotein receptor. J Biol Chem 272: $31700-$ 31706. 


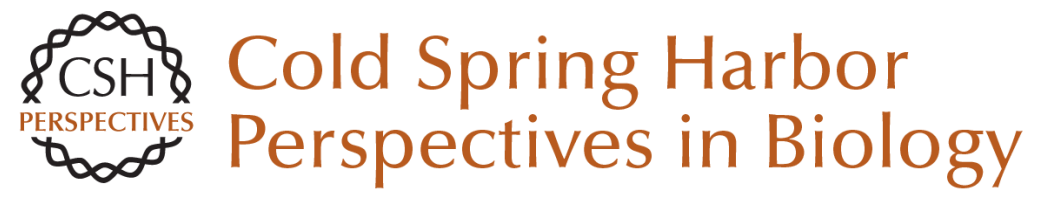

\section{Role of Endosomes and Lysosomes in Human Disease}

Frederick R. Maxfield

Cold Spring Harb Perspect Biol 2014; doi: 10.1101/cshperspect.a016931

Subject Collection Endocytosis

Endocytosis: Past, Present, and Future Sandra L. Schmid, Alexander Sorkin and Marino Zerial

Rab Proteins and the Compartmentalization of the Endosomal System Angela Wandinger-Ness and Marino Zerial

Cargo Sorting in the Endocytic Pathway: A Key Regulator of Cell Polarity and Tissue Dynamics Suzanne Eaton and Fernando Martin-Belmonte

Unconventional Functions for Clathrin, ESCRTs, and Other Endocytic Regulators in the Cytoskeleton, Cell Cycle, Nucleus, and Beyond: Links to Human Disease

Frances M. Brodsky, R. Thomas Sosa, Joel A. Ybe, et al.

Endocytosis of Viruses and Bacteria Pascale Cossart and Ari Helenius

Lysosomal Adaptation: How the Lysosome Responds to External Cues Carmine Settembre and Andrea Ballabio

Reciprocal Regulation of Endocytosis and Metabolism

Costin N. Antonescu, Timothy E. McGraw and Amira Klip

Endocytosis and Autophagy: Exploitation or Cooperation?

Sharon A. Tooze, Adi Abada and Zvulun Elazar
Imaging and Modeling the Dynamics of

Clathrin-Mediated Endocytosis Marcel Mettlen and Gaudenz Danuser

Endocytic Accessory Factors and Regulation of Clathrin-Mediated Endocytosis

Christien J. Merrifield and Marko Kaksonen

The Complex Ultrastructure of the Endolysosomal System Judith Klumperman and Graça Raposo

The Biogenesis of Lysosomes and Lysosome-Related Organelles J. Paul Luzio, Yvonne Hackmann, Nele M.G. Dieckmann, et al.

Endocytosis, Signaling, and Beyond Pier Paolo Di Fiore and Mark von Zastrow

Clathrin-Independent Pathways of Endocytosis Satyajit Mayor, Robert G. Parton and Julie G. Donaldson

The Role of Endocytosis during Morphogenetic Signaling Marcos Gonzalez-Gaitan and Frank Jülicher

Role of Endosomes and Lysosomes in Human Disease

Frederick R. Maxfield

For additional articles in this collection, see http://cshperspectives.cshlp.org/cgi/collection/

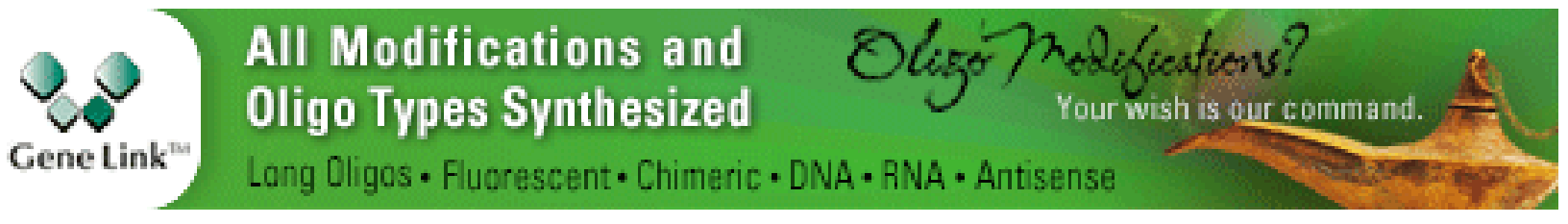


For additional articles in this collection, see http://cshperspectives.cshlp.org/cgi/collection/

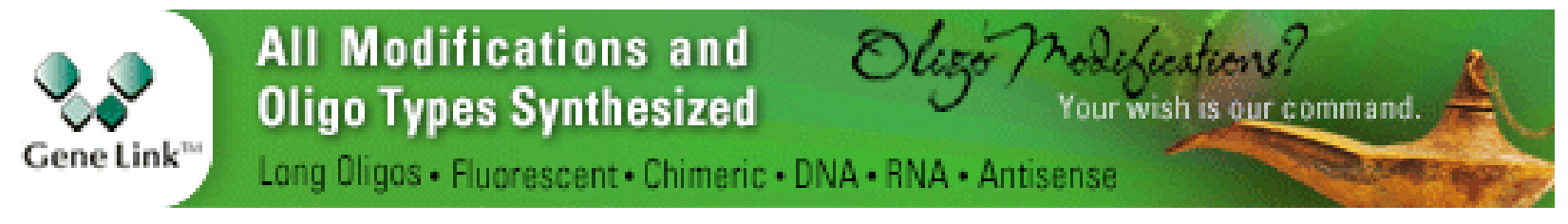

Copyright @ 2014 Cold Spring Harbor Laboratory Press; all rights reserved 